\title{
Sexual selection enables long-term coexistence despite ecological equivalence
}

Leithen K. M'Gonigle

Rupert Mazzucco

Sarah P. Otto

Ulf Dieckmann (dieckmann@iiasa.ac.at)

\section{Approved by}

Pavel Kabat

Director General and Chief Executive Officer

February 2015 
Running head: SEXUAL SELECTION AND COEXISTENCE

\title{
Sexual selection enables long-term coexistence despite ecological equivalence
}

\author{
Leithen K. M'Gonigle ${ }^{1, *}$, Rupert Mazzucco ${ }^{2}$, Sarah P. Otto ${ }^{1}$, \\ and Ulf Dieckmann ${ }^{2}$
}

1.

Department of Zoology, University of British Columbia

Vancouver, BC

Canada

V6T $1 Z 4$

2.

Evolution and Ecology Program

International Institute for Applied Systems Analysis

Laxenburg

Austria

1 * Corresponding author: Leithen K. M'Gonigle

2 e-mail: mgonigle@zoology.ubc.ca

3 phone: 1-510-423-1334 
Empirical data indicate that sexual preferences are critical for maintaining species boundaries ${ }^{1-4}$, yet theoretical work has suggested they can play only a minimal role in maintaining biodiversity on their own $^{5-9}$. This is because long-term coexistence within overlapping ranges is thought to be unlikely in the absence of ecological differentiation ${ }^{9}$. Here we challenge this widely held view by generalizing a standard model of sexual selection to include two ubiquitous features of populations with sexual selection: spatial variation in local carrying capacity and mate-search costs in females. We show that, when these two features are combined, sexual preferences can single-handedly maintain coexistence, even when spatial variation in local carrying capacity is so slight that it might go unnoticed empirically. This is the first theoretical study to demonstrate that sexual selection alone can promote the long-term coexistence of ecologically equivalent species with overlapping ranges, and it thus provides a novel explanation for the maintenance of species diversity.

A central objective of evolutionary ecology is to understand the mechanisms that allow species coexistence. One such mechanism is ecological differentiation. By occupying different niches, species in overlapping ranges are able to reduce direct competition among one another ${ }^{10}$. While there are numerous examples of closely related species occupying different ecological niches, many recently diverged and coexisting taxa are known to differ most dramatically in their secondary sexual characters, exhibiting few, if any, ecological differences ${ }^{1-4}$. It seems, therefore, that sexual selection is an important mechanism for maintaining coexistence. Indeed, models of sexual selection have shown that populations of choosy females and their preferred males can arise and, under various conditions, form reproductively isolated mating groups ${ }^{11-15}$. However, because sexual selection does not lead to ecological differentiation, species differing only in their mating preferences compete for the same ecological niche. This has traditionally led to the conclusion that, if their ranges overlap, one of these species 
will eventually displace the other ${ }^{5-9}$

Coexistence is facilitated by mechanisms that reduce range overlap among species. Sexual selection provides one such mechanism. Any process that creates spatial variation in female preferences indirectly also creates selection on male display traits, locally favouring those males that are most preferred by the local females. As a consequence, spatially segregated mating domains, characterized by the co-occurrence of matching display and preference traits, can emerge from populations with an initially random spatial distribution. Once segregated, interactions between different mating types are limited to individuals at the peripheries of these domains. In finite populations, however, the mating domains may shrink or grow, and the interface between them may drift randomly in space. Such fluctuations eventually lead to one mating domain replacing all others (Fig. 1a, c). In a pioneering study, Payne and Krakauer ${ }^{16}$ argued that lower dispersal in males with better mating prospects facilitates spatial segregation and maintains coexistence. In finite populations, however, such mating-dependent dispersal fails to stabilize long-term coexistence (Fig. S3). Given these difficulties associated with sexual selection, a recent review concluded that sexually divergent, but ecologically equivalent, species cannot coexist for significant lengths of time ${ }^{9}$.

Here we report model results that suggest the contrary and demonstrate that sexual selection can promote long-term coexistence, even without any ecological differentiation. Building on a standard model of sexual selection ${ }^{14}$, we develop an individual-based model to examine the long-term fate of species differing only in their secondary sexual characters in an ecologically neutral context with finite population sizes (details in Supplementary Information, SI). We assume a simple genetic structure with two unlinked haploid loci: the first locus (with alleles $Q$ and $q$ ) governs a display trait that is expressed only in males, while the second (with alleles $P$ and $p$ ) governs a 
preference trait that is expressed only in females (more than two alleles and quantitative mating traits are considered in the SI and Fig. 4c-d). Because we are interested in coexistence, and not speciation, we assume that the genetic variation at both loci is already present, for example, due to recent migration from allopatric ranges. All else being equal, females bearing a $P(p)$ allele prefer ${ }^{14-16}$ to mate with males carrying a $Q(q)$ allele by a factor $\alpha$, and a female's preference for a given male attenuates with increasing distance between them. Likewise, competition decreases as the distance between individuals increases. Competition is assumed to reduce an individual's probability of surviving until reproductive maturity (similar results are obtained if competition reduces fecundity, Fig. S4). Importantly, hybrids suffer no intrinsic fitness costs, other than potentially carrying mismatched preference and trait alleles.

Mating domains can be lost either through movement of the interface between them, or when individuals of one mating type colonize the domain of another mating type. In particular, because selection at the preference locus disappears when there is no variation at the display locus, foreign preference alleles may drift into regions with low variation in male display alleles, eventually causing displacement. Loss of mating domains can, however, be prevented by including two features ubiquitous in populations experiencing sexual selection: spatial variation in local carrying capacity and mate-search costs in females. Spatial variation in carrying capacity is present in most, if not all, biological systems (see Figs. 1 and 4 and the SI for model details). Mate-search costs occur if a female spends time and energy looking for a suitable mate and rejecting non-preferred males, thereby reducing her ability to invest in offspring. To account for such costs we assume that the fecundity of a particular female increases from 0 to a maximum level with the local density of available males, weighted according to her preference (SI). 
Our model confirms the longstanding view that sexual selection in homogeneous spatial models, without mate-search costs, does not facilitate coexistence and can, in fact, hasten the loss of diversity (compare Fig. 2a to 2b). Spatial variation in local carrying capacity, on its own, also has little, if any, effect in stabilizing populations (compare Fig. $2 \mathrm{~b}$ to $2 \mathrm{c}$ ). Sexual selection with mate-search costs slightly prolongs coexistence in a spatially homogeneous environment by helping to prevent mixing of the mating domains, but this effect is weak (Fig. 2d). However, in an environment with spatial variation in local carrying capacity, sexual selection with mate-search costs dramatically increases coexistence times (compare Fig. 2e to Fig. 2b and also Fig. 1a, c to Fig. 1b, d). In this case, mate-search costs curb the neutral drift of preference alleles, thus preventing the dilution of mating domains, while areas of high local carrying capacity provide spatial "anchors", stabilizing the location and size of these domains (Fig. 1b, d).

While neither spatial variation in local carrying capacity nor mate-search costs suffice on their own to stabilize populations, surprisingly little of both can be enough to ensure the long-term persistence of divergent mating types (Fig. 3). When mate-search costs in females are high, long-term coexistence can be maintained with less than $20 \%$ spatial variation in local carrying capacity. When mate-search costs are low, 50\% spatial variation in local carrying capacity is sufficient to stabilize mating domains. Throughout, we have kept population sizes relatively small, so as to exacerbate the challenge of coexistence in finite populations. When population sizes are larger, we find that as little as $10 \%$ variation in local carrying capacity suffices to stabilize mating domains (Fig. S5d). Levels of variation in this range may be difficult to detect in nature, especially if they are to be inferred from observing the stochastic spatial distribution of individuals. The stabilizing effect of spatial variation in local carrying capacity and mate-search costs readily extends to more realistic and natural landscapes (Fig. 4) and also to multiple 
genotypes (Fig. 4c-d) . As long as spatial variation in local carrying capacity does not become so insignificant that it hardly affects the landscape, or so asymmetric that a single local population dominates, different mating domains are maintained in mosaic sympatry ${ }^{17,18}$ (Fig. S7). Our findings are also robust to changes in female-preference strength, mate-search distance, movement distance, and competition distance (Figs. S5a, S6), to changes in the relative importance of ecological competition versus sexual selection (Fig. S5b-c), to changes in the genetic architecture of the display and preference traits (Fig. S8), and to including selective differences between male display traits (Fig. S9). Generally, long-term coexistence occurs if female preferences are sufficiently strong to prevent extensive interbreeding, and if individuals move and interact on a spatial scale such that they are affected by spatial variation in local carrying capacity. This phenomenon can be interpreted more generally: whenever positive frequency dependence creates multiple stable states, global coexistence of these states becomes possible in a spatially structured environment if this structure allows the domains in which those states are realized to become anchored in space. In this vein, our results in Fig. 4 extend a previous finding from theoretical work on hybrid zones, predicting that the spatial interface between species moves in space until settling in a region of low population density ${ }^{19,20}$. Similarly, earlier theoretical work ${ }^{21}$ using habitat boundaries for anchoring mating-domains, has shown that ecologically equivalent types can coexist when fecundity drops, or mortality or mobility rise, in the company of heterospecifics.

Because both spatial variation in local carrying capacity and costs associated with mate search are ubiquitous in nature, our model may provide an explanation for the coexistence of many species whose reproductive barriers primarily involve mating preferences. For example, local habitat availability and quality vary around the shoreline of Lake Victoria ${ }^{22}$. The mechanism reported here could help explain how ecologically similar cichlid species can coexist in such vast diversity. That sexual differences have 
been a primary force maintaining cichlid species' boundaries is supported by the increasing frequency of hybridization that is occurring as a consequence of high turbidity levels, which reduce a female's ability to discern male phenotypes ${ }^{22}$. Similar explanations could plausibly be applied to other species that seem to be largely maintained by sexual selection (e.g., species of fruit flies ${ }^{23}$, weakly electric fish ${ }^{24}$, frogs ${ }^{25}$, crickets $^{3}$, and grasshoppers ${ }^{26}$, among others). To test this hypothesis, one could analyse spatial associations between mating domains and local carrying capacity: Fig. 4 suggests that boundaries of mating domains often align with troughs of low local carrying capacity.

Our work demonstrates that, with variation in local carrying capacity over space and costs to females that encounter few preferred mates, sexual selection can maintain species that are not ecologically differentiated. This is in stark contrast to the widespread opinion that sexual selection, on its own, is unable to maintain ecologically equivalent species that overlap in space. Throughout, we have deliberately avoided making any claims about the emergence of diversity and speciation, choosing instead to focus on the coexistence of mating types. Further theoretical work is, therefore, needed to determine which conditions are most conducive to the initial appearance of multiple mating types, and further empirical work is needed to show how the mechanism presented here helps explain natural patterns of coexistence and diversity.

\section{Methods Summary}

We develop an individual-based model of sexual selection ${ }^{14}$ in a spatially explicit ecological framework. Individuals are distributed across a continuous habitat in one or two dimensions with wrap-around boundaries. All individuals compete for resources, 
whose density at any location is given by a local carrying capacity. Except where noted, the local carrying capacity exhibits two peaks, each of the same Gaussian shape. Competition reduces an individual's resource share, and thereby its survival probability, with the competitive impact of other individuals decreasing with distance according to a Gaussian function. Surviving females encounter surviving males with a probability decreasing with distance according to a Gaussian function, and females choose mates based on their preferences for the male's displays. After mating, females produce offspring in proportion to their fecundities, which are lower for females who experienced higher mate-search costs. After producing offspring, the parents die and the offspring move a distance drawn from a Gaussian function in a direction chosen at random. While the female preference trait and the male display trait are genetically based (each being determined by a diallelic locus, except where noted), there are no genetic differences in ecological function or competitive ability among individuals, which are, therefore, all ecologically equivalent. See SI for complete model details and for information about alternative models explored to examine the robustness of our results.

\section{Acknowledgements}

The authors would like to thank J. S. Brown, R. G. FitzJohn, D. E. Irwin, J. Ohlberger, J. L. Payne, A. Pomiankowski, G. S. van Doorn, and two anonymous reviewers for providing valuable feedback. Funding was provided by a Natural Sciences and Engineering Research Council (Canada) grant to L.K.M. (CGS-D) and S.P.O. (Discovery Grant). L.K.M. received additional support from the European Science Foundation Research Networking Programme "Frontiers of Speciation Research". R.M. and U.D. gratefully acknowledge support by the Vienna Science and Technology Fund (WWTF). U.D. 
received additional financial support from the European Commission, the European Science Foundation, the Austrian Science Fund, and the Austrian Ministry of Science and Research.

\section{Author Contributions}

U.D. and L.K.M. conceived this project. L.K.M., R.M., S.P.O., and U.D. discussed and designed the model. L.K.M. implemented the model with input from R.M., analysed the results together with R.M., S.P.O., and U.D. and prepared the manuscript. L.K.M., R.M., S.P.O., and U.D. jointly edited the manuscript.

\section{Author Information}

Simulation code is available at www.zoology.ubc.ca/prog/coexist. Correspondence and requests for materials should be addressed to L.K.M. (mgonigle@zoology.ubc.ca).

\section{References}

[1] Eberhard, W. G. Sexual Selection and Animal Genitalia. Harvard University Press, Cambridge, Mass., (1985).

[2] Seehausen, O. and van Alphen, J. J. M. Can sympatric speciation by disruptive sexual selection explain rapid evolution of cichlid diversity in Lake Victoria? Ecol. Lett. 2, 262-271 (1999).

[3] Gray, D. A. and Cade, W. H. Sexual selection and speciation in field crickets. Proc. Natl. Acad. Sci. USA 97, 14449-14454 (2000). 
[4] Wilson, A. B., Noack-Kunnmann, K., and Meyer, A. Incipient speciation in sympatric Nicaraguan crater lake fishes: sexual selection versus ecological diversification. Proc. R. Soc. Lond. B 267, 2133-2141 (2000).

[5] Turner, G. F. and Burrows, M. T. A model of sympatric speciation by sexual selection. Proc. R. Soc. Lond. B 260, 287-292 (1995).

[6] Panhuis, T. M., Butlin, R., Zuk, M., and Tregenza, T. Sexual selection and speciation. Trends Ecol. Evol. 16, 364-371 (2001).

[7] van Doorn, G. S., Dieckmann, U., and Weissing, F. J. Sympatric speciation by sexual selection: a critical reevaluation. Am. Nat. 163, 709-725 (2004).

[8] Johansson, J. and Ripa, J. Will sympatric speciation fail due to stochastic competitive exclusion? Am. Nat. 168, 572-578 (2006).

[9] Weissing, F. J., Edelaar, P., and van Doorn, G. S. Adaptive speciation theory: a conceptual review. Behav. Ecol. Sociobiol. 65, 461-480 (2011).

[10] Schluter, D. The Ecology of Adaptive Radiation. Oxford University Press, (2000).

[11] Fisher, R. A. The Genetical Theory of Natural Selection. Oxford: Clarendon Press, 1st edition, (1930).

[12] Lande, R. Models of speciation by sexual selection on polygenic traits. Proc. Natl. Acad. Sci. USA 78, 3721-3725 (1981).

[13] Lande, R. Rapid origin of sexual isolation and character divergence in a cline. Evolution 36, 213-223 (1982).

[14] Kirkpatrick, M. Sexual selection and the evolution of female choice. Evolution 36, $1-12(1982)$. 
[15] Seger, J. Unifying genetic models for the evolution of female choice. Evolution 39, 1185-1193 (1985).

[16] Payne, R. J. H. and Krakauer, D. C. Sexual selection, space, and speciation. Evolution 51, 1-9 (1997).

[17] Mallet, J. Hybridization, ecological races, and the nature of species: empirical evidence for the ease of speciation. Phil. Trans. R. Soc. B 363, 2971-2986 (2008).

[18] Mallet, J., Meyer, A., Nosil, P., and Feder, J. L. Space, sympatry and speciation. J. Evol. Bio. 22, 2332-2341 (2009).

[19] Barton, N. H. and Hewitt, G. M. Analysis of hybrid zones. Ann. Rev. Ecol. Syst. 16, 113-148 (1985).

[20] Barton, N. H. and Hewitt, G. M. Adaptation, speciation and hybrid zones. Nature 341, 497-503 (1989).

[21] Dieckmann, U. Assortative mating and spatial coexistence. In Adaptive Speciation, Dieckmann, U., Metz, J. A. J., Doebeli, M., and Tautz, D., editors, 306-307. Cambridge University Press (2004).

[22] Seehausen, O., van Alphen, J. J. M., and Witte, F. Cichlid fish diversity threatened by eutrophication that curbs sexual selection. Science 277, 1808-1809 (1997).

[23] Hollocher, H., Ting, C., Pollack, F., and Wu, C. Incipient speciation by sexual isolation in Drosophila melanogaster: variation in mating preference and correlation between sexes. Evolution 51, 1175-1181 (1997).

[24] Feulner, P. G. D., Kirschbaum, F., and Tiedemann, R. Adaptive radiation in the Congo River: an ecological speciation scenario for African weakly electric fish (Teleostei; Mormyridae; Campylomormyrus). J. Physiol. Paris 102, 340-346 (2008). 
[25] Ryan, M. J. and Wilczynski, W. Coevolution of sender and receiver: effects of local mate preference in cricket frogs. Science 240, 1786-1788 (1988).

[26] Tregenza, T., Pritchard, V. L., and Butlin, R. K. The origins of premating reproductive isolation: testing hypotheses in the grasshopper Chorthippus parallelus. Evolution 54, 1687-1698 (2000). 

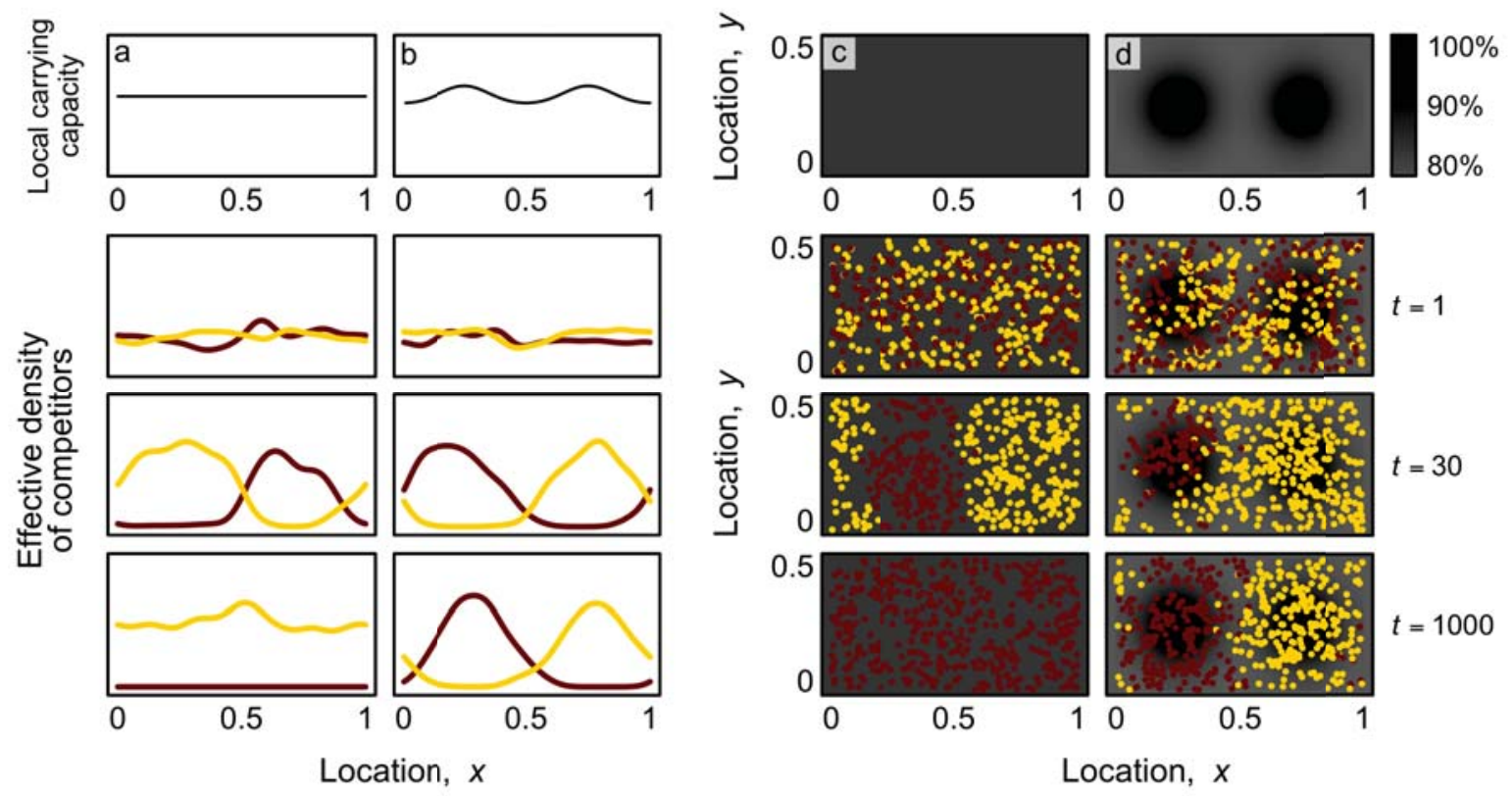

Figure 1: Sexual selection enables long-term coexistence of ecologically equivalent species. We consider a population distributed across a continuous habitat in one dimension (columns $\mathbf{a}, \mathbf{b}$ ) or two dimensions (columns $\mathbf{c}, \mathbf{d}$ ) with a local carrying capacity that is either spatially uniform (a, c: top panels) or that exhibits two peaks (b, d: top panels). Each peak is of Gaussian shape with standard deviation $\sigma_{\mathrm{k}}$. The level $v$ of spatial variation may be altered by changing the height of these peaks relative to the troughs between them. A value of $v=0.25$, as in $\mathbf{b}$ and $\mathbf{d}$, means that local carrying capacity at the peaks is elevated by $25 \%$. The three lower rows show model runs through time. Each generation, individuals survive after a round of local competition and reproduce after a round of local mating, followed by offspring movement and the death of all parents. Competition between individuals decreases with their distance according to a Gaussian function with standard deviation $\sigma_{\mathrm{s}}$. Coloured curves in $\mathbf{a}$ and $\mathbf{b}$ show the effective local density of competitors of each type (weighted by their competitive effect, SI, Eq. 4), while dots in $\mathbf{c}$ and $\mathbf{d}$ show surviving adults. Individuals are coloured according to their display locus genotype (similar patterns are observed at the preference locus; Fig. S2). Females are $\alpha$ times more likely to mate with a preferred male, when encountered. Males are encountered with a probability that decreases with the distance between them and the female according to a Gaussian function with standard deviation $\sigma_{\mathrm{f}}$. Female fecundity declines with the strength of mate-search costs $m$. Movement distances are drawn from a Gaussian function with standard deviation $\sigma_{\mathrm{m}}$, centered at 0 , with wrap-around boundaries. The total carrying capacity is $K=500$, supporting the survival of approximately half of the $N=1000$ offspring produced each generation; other parameters: $\sigma_{\mathrm{k}}=0.1, \sigma_{\mathrm{s}}=0.05$, $\alpha=5, \sigma_{\mathrm{f}}=0.05, \sigma_{\mathrm{m}}=0.05$, and $m / K=1$ (roughly halving fecundity, Fig. S1). 

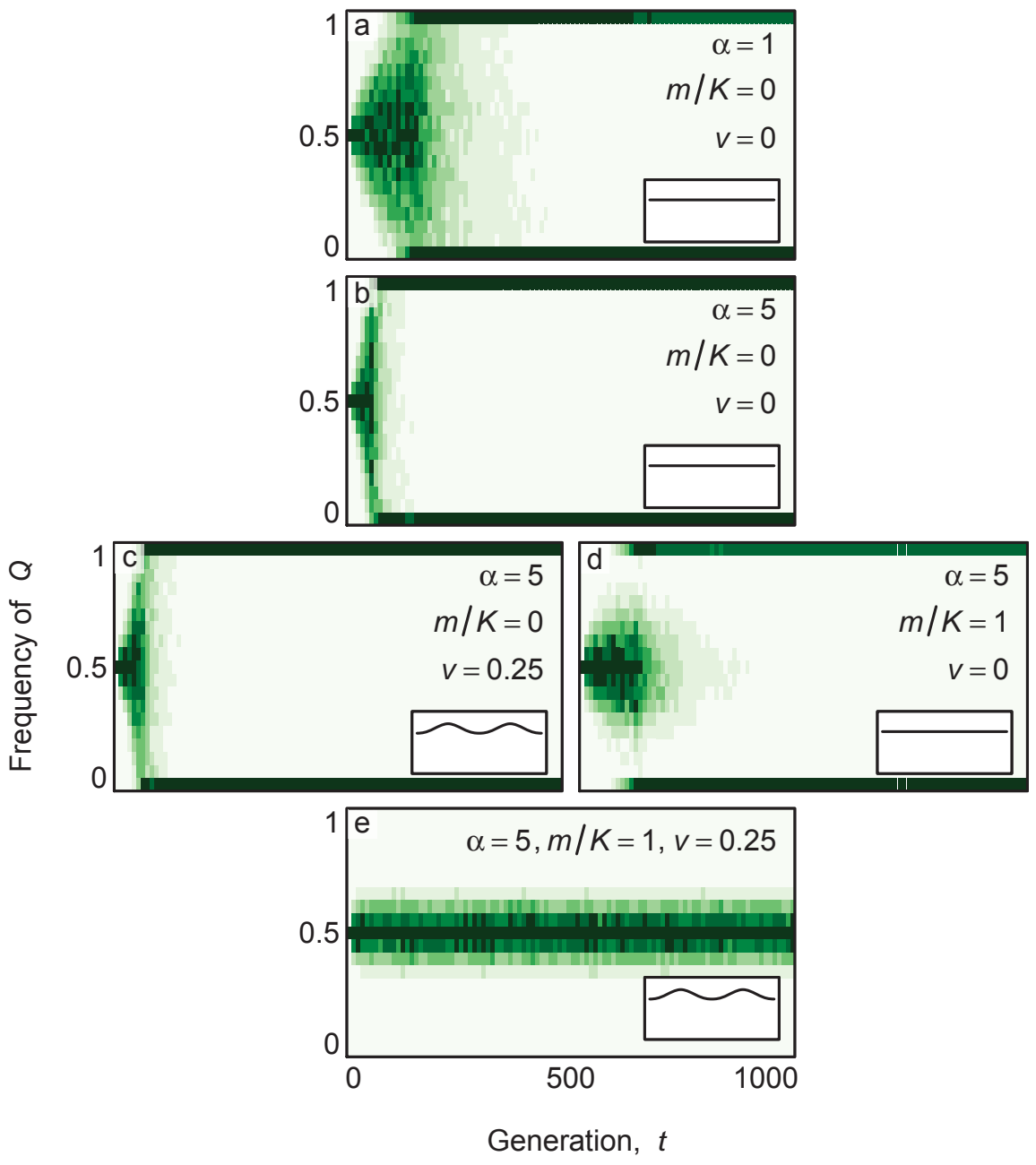

Figure 2: Conditions for long-term coexistence. Panels show distributions of allele frequencies at the display locus through time across 1000 model runs in a two-dimensional landscape; coexistence occurs only while these frequencies remain intermediate. Inset panels depict the spatial variation in local carrying capacity as viewed along transects at $y=0.25$. a Homogeneous environment with no sexual selection $(\alpha=1)$. $\mathbf{b}$. Same as a, except that females are choosy $(\alpha=5)$. c. Same as $b$, except with variation in local carrying capacity $(v=0.25)$. d. Same as $b$, except with mate-search costs in females $(m / K=1)$. e. Same as $b$, except with spatial variation in local carrying capacity $(v=0.25)$ and matesearch costs in females $(m / K=1)$; only when both features are combined is long-term coexistence observed. To focus on the maintenance of coexistence, we begin with two equally sized and spatially segregated populations of $P Q$ and $p q$ genotypes (all individuals on the left half of the arena initially have the $P Q$ genotype, while all individuals on the right initially have the $p q$ genotype). This mimics a scenario in which types that previously arose in allopatry come back into contact, revealing the conditions under which they can persist in sympatry. All other parameters are as in Fig. 1. 


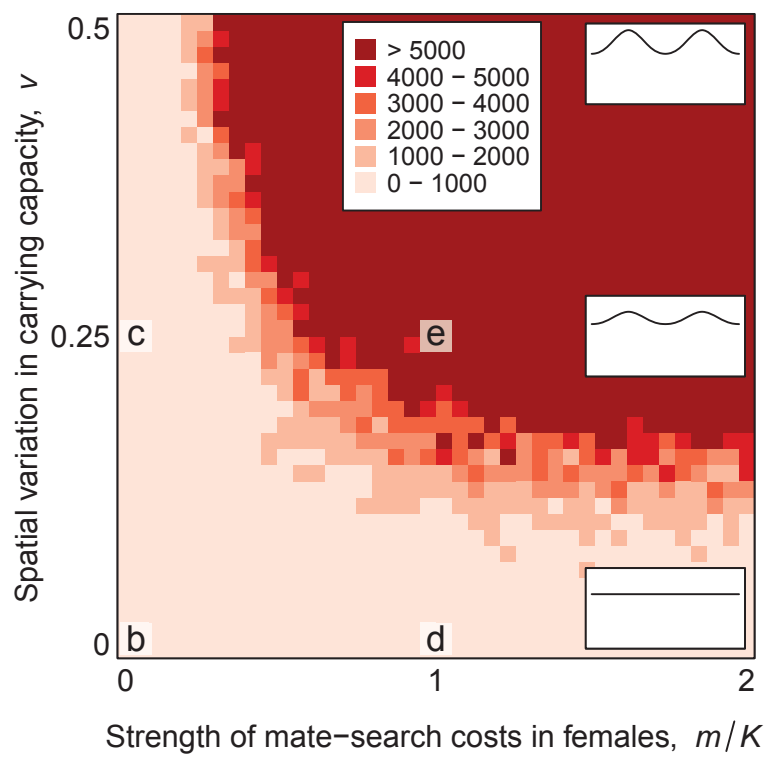

Figure 3: Conditions for long-term coexistence. Shading indicates the number of generations that polymorphism at the display locus persists when females are choosy $(\alpha=5)$ in a two-dimensional landscape (darker $=$ longer). Each cell represents the mean time to loss of polymorphism for 10 replicate model runs. Letters indicate parameter combinations used to generate the lower four panels in Fig. 2. Inset panels illustrate the extent of spatial variation in local carrying capacity for the three parameter values shown along the vertical axis. Model runs are initialized as in Fig. 2. All other parameters are as in Fig. 1. 


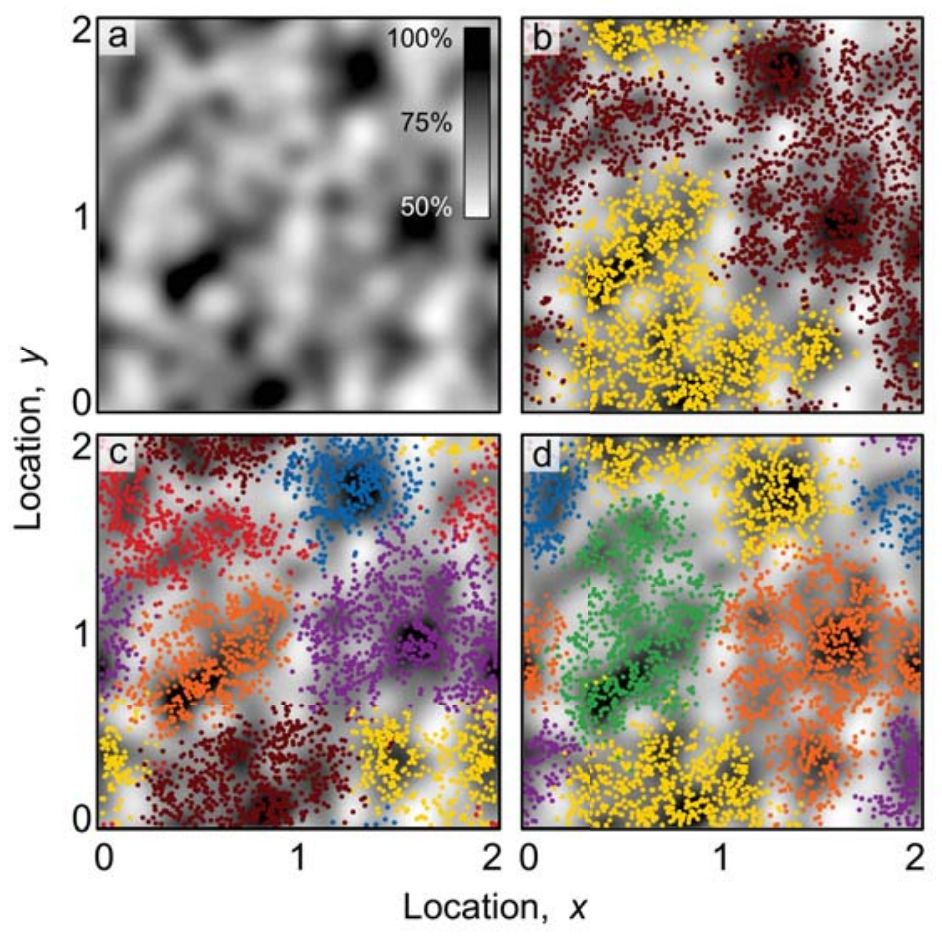

Figure 4: Mosaic sympatry. Four representative model runs in a patchy two-dimensional landscape with random variation in local carrying capacity. Panel a depicts the underlying spatial variation in local carrying capacity, while panels $\mathbf{b}-\mathbf{d}$ show results from independent model runs after 10,000 generations overlaid on the local carrying capacity. Panel $\mathbf{b}$ is initialized with two types, whereas panels $\mathbf{c}$ and $\mathbf{d}$ are initialized with ten display alleles and ten corresponding preference alleles, all at equal frequencies and distributed randomly across the arena (SI, Section S2.2). Some of these alleles are then lost during the colonization phase. As in Fig. 1, individuals are coloured according to their genotype at the display locus. The spatial arena is eight times larger than in Fig. 1 and the total carrying capacity is $K=4000$, supporting the survival of approximately half of the $N=8000$ offspring produced each generation. All other parameters are as in Fig. 1 (except for $v$, which is defined specifically for bimodal landscapes); for comparison, the coefficient of variation in local carrying capacity is 0.125 here, compared with 0.066 in Fig. 1d. 


\section{Supplementary Information}

\section{S1 Model description}

We consider an individual-based model with discrete non-overlapping generations in one- or two-dimensional continuous space with wrap-around boundaries. Below, we describe the two-dimensional model, from which the corresponding one-dimensional model is readily generated by removing the spatial $y$-dimension. Each individual has a spatial location and is characterized by a display trait (expressed only in males) and a preference trait (expressed only in females). In our main set of model runs, these traits are assumed to be governed by separate unlinked haploid loci, each with two alleles (display alleles are denoted by $Q / q$ and preference alleles by $P / p$ ). Each generation, $N$ individuals are produced and compete for resources, with those experiencing stronger competition being more likely to die before reaching reproductive maturity. Resources in our model may be interpreted in the broadest possible sense, describing the biotic and abiotic factors that are subject to local ecological competition. Among the individuals surviving ecological competition, females choose mates, with the probability of a specific male being chosen depending on her mating preference and the spatial distance separating them. Females produce offspring in proportion to their fecundities. Offspring then disperse from their natal location and the parents die. Below we detail these steps in the order in which they occur. The names and descriptions of all parameters and variables are listed in Table S1. 


\section{S1.1 Competition for resources}

The habitat at each location $(x, y)$ is characterized by the local density $k(x, y)$ of available resources. The total amount of resources over the spatial arena is given by $K=\iint k(x, y) \mathrm{d} x \mathrm{~d} y$. The function relating resource gain to survival is chosen such that if every individual received an equal share of these resources, the expected number of survivors would be $K$. Consequently, we refer to $k(x, y)$ as the local carrying capacity and to $K$ as the total carrying capacity. Except for Figs. 4 and S7, we investigate a local carrying capacity that is symmetrically bimodal, with two peaks located at $(x, y)=(0.25,0.25)$ and $(x, y)=(0.75,0.25)$. If we considered only these two focal Gaussians, the resource availability would not be symmetric about the peaks. To avoid such an asymmetry, we constructed a periodic landscape given by

$$
k(x, y)=\left(b+\sum_{i, j} \exp \left(-\frac{(x-(0.25+i / 2))^{2}+(y-(0.25+j / 2))^{2}}{2 \sigma_{\mathrm{k}}^{2}}\right)\right) k_{0},
$$

for $x$ in $[0,1]$ and $y$ in $[0,0.5]$, where the sum is taken over all pairs of integers, and where $\sigma_{\mathrm{k}}$ denotes the widths of the Gaussian peaks. The parameters $b$ and $k_{0}$ allow us to adjust the average height and degree of variation in $k(x, y)$. Specifically, the height is adjusted such that the total carrying capacity equals $K$, and the degree of variation is adjusted to give the desired relation between peaks and troughs. For the local carrying capacity in Eq. (1), it is natural, for easy comparison between the one-dimensional and the two-dimensional model, to measure the degree of spatial variation along the transect spanning both peaks as

$$
v=\frac{\max k(x, y)-\min k(x, y)}{\min k(x, y)} .
$$

A value of $v=0.25$ therefore means that the local carrying capacity is $25 \%$ higher at the peaks than at the troughs between them. For Fig. S7, landscapes are generated in a 
similar way, except that the heights and widths of the two peaks differ. For Fig. 4 , the landscape is generated by adding white noise to the baseline level, filtered to have a reasonable amount of spatial autocorrelation, with the highest peak set to twice the height of the lowest trough.

Through competition, each individual obtains a share of the local carrying capacity, which we refer to as its resource share,

$$
\rho_{i}=\frac{k\left(x_{i}, y_{i}\right)}{\sum_{j} n_{i j}}
$$

where $n_{i j}$ is the contribution of individual $j$ to the effective density of competitors at the location of individual $i$, and the sum extends over all $N$ individuals. The competitive impact of individual $j$ on individual $i$ decreases with the distance $d_{i j}$ separating them, according to a Gaussian function with standard deviation $\sigma_{\mathrm{s}}$

$$
n_{i j}=\exp \left(-d_{i j}^{2} /\left(2 \sigma_{\mathrm{s}}^{2}\right)\right) /\left(2 \pi \sigma_{\mathrm{s}}^{2}\right)
$$

in the one-dimensional model, the divisor is $\sqrt{2 \pi} \sigma_{\mathrm{s}}$. Note that the effect $n_{i i}$ of an individual $i$ on itself declines as $\sigma_{\mathrm{s}}$ increases, because the individual then competes for resources over larger distances and thus has less of a negative impact on its available resources.

As defined, the resource share of an individual $i$ is approximately $K / N$. This can be seen by assuming that the $N$ individuals in the population are distributed over the arena according to the local carrying capacity, so that their expected density is $N k(x, y) / K$. 
Replacing the sum over individuals in Eq. 3 with an integral over space, we obtain

$$
\begin{aligned}
\rho_{i} & =\frac{k\left(x_{i}, y_{i}\right)}{\iint \frac{N k(x, y)}{K} \frac{\exp \left(-\left(\left(x_{i}-x\right)^{2}+\left(y_{i}-y\right)^{2}\right) /\left(2 \sigma_{\mathrm{s}}^{2}\right)\right)}{2 \pi \sigma_{\mathrm{s}}^{2}} \mathrm{~d} x \mathrm{~d} y} \\
& =K / N+O(v),
\end{aligned}
$$

where the second line assumes that spatial variation in the local carrying capacity is low. In our individual-based model runs, departures from the above occur due to clumping, fecundity variation over space (Section S1.4), as well as discrepancies due to replacing the sum in Eq. 3 with the integral in Eq. 5 (especially when $\sigma_{\mathrm{s}}$ is very small or large relative to the arena). That said, the mean resource share is typically close to $K / N$ in our model runs.

In Fig. S1 we show the effect of spatial variation in local carrying capacity $k\left(x_{i}, y_{i}\right)$ on various components of fitness, including the resource share $\rho_{i}$. Interestingly, ecological competition is weaker $\left(\rho_{i}\right.$ is higher) in regions of low carrying capacity (Fig. S1a), increasing the survival probability $s_{i}$ of individuals in these regions (Section S1.2 and Fig. S1b). This occurs because females are less likely to encounter preferred males wherever the carrying capacity is low, causing their fecundity to be lower due to increased mate-search costs $c_{i}$ (Section S1.4 and Fig. S1c). Consequently, fewer offspring are produced than expected based on the low local carrying capacity, resulting in weaker competition among those offspring. The net result of lower ecological competition and higher mate-search costs in regions with low local carrying capacity is that females have roughly equal fitness across space. 


\section{S1.2 Survival}

We assume that individuals that gain more resources are more likely to survive to reproductive maturity. The probability $s_{i}$ of such survival is assumed to be zero when an individual fails to gain any resources, to rise approximately linearly with its resource share $\rho_{i}$ when that share is small, and to taper off at a maximal survival probability of $s_{\max }$ (ranging between 0 and 1). Specifically, we use a hyperbolic (or Holling type-2) function ${ }^{1}$ to relate resource share to the probability of survival,

$$
s_{i}=\frac{s_{\max }}{1+r / \rho_{i}},
$$

where $r$ is the resource share that must be obtained for an individual to survive with a probability equal to half the maximal survival probability. Unless stated otherwise, we assume that the maximum probability $s_{\max }$ of surviving to reproductive maturity equals 1.

The value of $r$ is chosen to ensure that, on average, $K$ individuals survive to reproduce if all individuals obtain an equal share of resources $\left(\rho_{i}=K / N\right)$. By setting the expected survival probability $s_{i}$ to $K / N$ in Eq. 6 and substituting $\rho_{i}=K / N$, we obtain $r=s_{\max }-K / N$. With this choice of $r$, approximately $K$ individuals survive each generation (with a variance that is typically small). For example, in Fig. S1, the average survival probability is 0.484 , close to the expected value of $K / N=0.5$. While competition for resources causes substantial mortality, survival probabilities across the arena differ only slightly (Fig. S1b). Importantly, the survival of an individual does not depend on whether or not it is a hybrid. 


\section{S1.3 Mating}

Of the individuals that survive to mate, the probability that female $i$ chooses male $j$ as a mate depends on whether his display trait matches her preference trait and on the spatial distance separating them. Females bearing a $P(p)$ allele prefer males bearing a $Q$ (q) allele by a factor $\alpha$. We assume that females encounter males in the vicinity of their home location. Specifically, each female spends a proportion of time at distance $d_{i j}$ from her home that is described by a Gaussian distribution with standard deviation $\sigma_{\mathrm{f}}$, so that her encounter probability $e_{i j}$ with a male at distance $d_{i j}$ is proportional to

$$
e_{i j}=\exp \left(-d_{i j}^{2} /\left(2 \sigma_{\mathrm{f}}^{2}\right)\right) /\left(2 \pi \sigma_{\mathrm{f}}^{2}\right) ;
$$

in the one-dimensional model, the divisor is $\sqrt{2 \pi} \sigma_{\mathrm{f}}$. In our main model, we assume that females encounter resources and males over the same spatial scales (i.e., $\sigma_{\mathrm{f}}=\sigma_{\mathrm{s}}$ ); we relax this assumption in Fig. S6. The probability that female $i$ chooses male $j$ as a mate is proportional to

$$
p_{i j}=\alpha^{\delta_{i j}-1} e_{i j}
$$

where $\delta_{i j}$ equals 1 when the display trait of male $j$ matches the preference trait of female $i$, and 0 otherwise. Once a female chooses a mate, we assume that all her offspring are sired by that male (monogamy).

\section{S1.4 Reproduction}

The fecundity of a female $i$ is given by:

$$
f_{i}=f_{\max }\left(1-c_{i}\right),
$$


where $f_{\max }$ is the maximum fecundity and $c_{i}$ (ranging from 0 to 1 ) measures the cost associated with finding a preferred mate for female $i$. The factor $1-c_{i}$ is assumed to be zero when there are no preferred males locally, to rise approximately linearly with the local density of preferred males,

$$
\mu_{i}=\sum_{\text {males }} p_{i j}
$$

and to taper off at 1 when preferred mates are readily encountered, resulting in maximal fecundity. Specifically, we use a hyperbolic (or Holling type-2) function ${ }^{2}$,

$$
1-c_{i}=\frac{1}{1+m / \mu_{i}}
$$

where $m$ is the value of $\mu_{i}$ at which a female's fecundity is halved by mate-search costs.

Because $\mu_{i}$ is obtained by summing over the entire male population, its value can be large, on the order of the number of surviving males, so values of $m$ on the order of the surviving population's size $K$ are needed for costs to be appreciable. This is why we express $m$ relative to $K$, specifying the ratio $m / K$ in the figures. We refer to $c_{i}$ as the mate-search cost of female $i$ and to $m$ as the strength of mate-search costs.

Unless noted otherwise, we use $m=500$. In our main set of model runs (with $m / K=1)$, mate-search costs reduce female fecundity by about $50 \%$, on average, from the maximum fecundity (Fig. S1c), with relatively minor differences in fecundity among females over space. Other values of $m$ are explored in Fig. 3. For $m=0$, all females have equal and maximal fecundity. As $m$ is raised, fecundity declines and becomes more variable, with females in low-density regions or surrounded by non-preferred males having lower fecundity (Fig. S2).

After mating, offspring are produced. Inheritance at both loci is Mendelian, and we assume no linkage between the display and preference loci, except where noted (Section 
S2.8). To allow us to explore various parameters relating to competition and mate-search costs independently, we hold the total number of offspring constant at $N$. For each offspring, a mother is chosen in proportion to the females' fecundities. Consequently, the maximum fecundity $f_{\max }$ only matters insofar as it is high enough to result in at least $N$ offspring being produced across the population. Similar patterns are observed when $f_{\max }$ is fixed and offspring numbers are given by a Poisson distribution with a mean of $f_{i}$ for each female (data not shown). We consider $N$ to be the total number of offspring surviving the phase during which resources are largely provided by the parents, after which the offspring move and begin the next phase of competition for resources.

\section{S1.5 Movement}

Each offspring moves from its mother's location according to a distance drawn from a Gaussian function with mean 0 and standard deviation $\sigma_{\mathrm{m}}$. Movements occur in all directions with equal probability.

\section{S2 Model extensions}

To assess the robustness of our results, we consider several extensions and/or modifications to our main model described above.

\section{S2.1 Allowing mating to impact dispersal}

To compare our results with those of Payne and Krakauer ${ }^{3}$, we consider mating-dependent dispersal. In their model, male movement distances are lower for males with better mating prospects, and we thus assume that the movement distance of 
male $j$ is drawn from a Gaussian function with mean 0 and standard deviation

$$
\sigma_{\mathrm{m}, j}=\sigma_{\mathrm{m}} \exp \left(-l \frac{\sum_{i} p_{i j}}{\sum_{i k} p_{i k}}\right)
$$

where $l$ determines how quickly movement distances decrease with increasing mating prospects and $p_{i j}$ is given by Eq. 8 in Section S1.3. For $l=0$, the above reduces to our main model. We find that the addition of mating-dependent dispersal in males extends coexistence times only marginally, if at all (compare Fig. S3a to S3b). We also examine the related case in which males with low mating prospects move farther, but again, coexistence times are not appreciably prolonged in our individual-based model.

\section{S2.2 Introducing multiple allelic types}

To examine whether long-term coexistence of more than two types is possible, we extend our main model so that one of $n$ alleles $p_{1}, \ldots, p_{n}$ can occur at the preference locus and one of $n$ alleles $q_{1}, \ldots, q_{n}$ can occur at the display locus. Specifically, in Fig. 4, we consider $n=10$ preference and display types. A female with preference allele $p_{i}$ prefers males with display allele $q_{i}$ to all other males by the factor $\alpha$. All other components of mate choice remain the same as for our main model with $n=2$ mating types.

\section{S2.3 Allowing competition to impact fecundity}

In our main model, competitive interactions reduce the survival probability of an individual. Alternatively, individuals that gain fewer resources might survive, but have lower fecundity. To explore this possibility, we allow all $N$ offspring to survive, while reducing their reproductive success according to the impact of competition, as measured by $s_{i}$. Specifically, for males, the probability of being chosen as a mate is set to 
$p_{i j}=\alpha^{\delta_{i j}-1} e_{i j} s_{i}$. Likewise for females, fecundity is set to $f_{i}=f_{\max }\left(1-c_{i}\right) s_{i}$. Such competition-dependent fecundity generates less demographic stochasticity, because all individuals reach reproductive maturity and can mate, albeit with reduced probability when their resource share $\rho_{i}$ is low. Indeed, all else being equal, incorporating competitive effects on fecundity, rather than survival, enables long-term coexistence over a wider range of parameters (compare Fig. S4 to Fig. 3).

\section{S2.4 Altering the strength of density-dependent competition}

We define the strength of density-dependent competition as

$$
\lambda=r /(1-K / N),
$$

with $r=s_{\max }-K / N$ (Section S1.2). In our main model, the maximum survival rate $s_{\max }$ is set to 1 so that $\lambda=1$, indicating that survival is strongly density-dependent. At the other extreme, if $s_{\max }$ is set to $K / N$, all individuals survive with probability $s_{\max }=K / N$, regardless of their resource share, so there is no density-dependent effect on survival $(\lambda=0)$. As shown in Fig. S5b, coexistence does not occur in the absence of density dependence $(\lambda=0)$; spatial variation in local carrying capacity then becomes irrelevant and cannot stabilize mating domains in space. As the importance of competition increases (larger $\lambda$, or equivalently, larger $s_{\max }$ ), long-term coexistence can occur over a wider range of parameters. Once about half of the mortality is due to density-dependent competition $(\lambda>0.5)$, results become similar to those for $\lambda=1$. 


\section{S2.5 Altering the impact of ecological competition}

We explore the impact of ecological competition by varying the expected survival probability $\bar{s}=K / N$ of offspring, while the total carrying capacity $K$ and the strength $\lambda$ of density-dependent competition are held constant (Fig. S5c). When the impact of ecological competition is small ( $\bar{s}$ near 1$)$, long-term coexistence requires much higher levels of spatial variation in local carrying capacity. Once ecological competition is sufficiently strong (removing at least $40 \%$ of offspring; $\bar{s}<0.6$ ), results become less sensitive to $\bar{s}$.

\section{S2.6 Altering the degree of demographic stochasticity}

If each of $N$ offspring survives with probability $\bar{s}$, the number of mating individuals follows a binomial distribution with mean $N \bar{s}$ and variance $N \bar{s}(1-\bar{s})$. The resultant coefficient of variation thus equals $\sqrt{1 / \bar{s}-1} / \sqrt{N}$, which grows as $\bar{s}$ shrinks. The associated rise in demographic stochasticity with smaller $\bar{s}$ may contribute to the slight rise in spatial variation in local carrying capacity required for maintaining long-term coexistence below $\bar{s}=0.5$ in Fig. S5c.

The effects of demographic stochasticity can also be seen in Fig. S5d, where the total carrying capacity $K$ is varied (together with the time point at which coexistence is evaluated, at generation $10 K)$, while the strength $\lambda$ of density-dependent competition and the expected survival probability $\bar{s}=K / N$ are held constant. Because we are interested in the effects of population size per se, we also keep constant the relative strength of mate-search costs $(m / K=1)$, so the ease with which females encounter preferred mates remains unaffected by variation in $K$. All else being equal, larger population sizes facilitate the long-term maintenance of coexisting types, as expected 
given the associated reduction in demographic stochasticity (the aforementioned coefficient of variation falls in proportion to $1 / \sqrt{N}$ ).

\section{S2.7 Altering the spatial scales of competition, mate search, and movement}

In our main model, we equate the spatial scales of three processes: competition $\left(\sigma_{\mathrm{s}}=0.05\right)$, mate search $\left(\sigma_{\mathrm{f}}=0.05\right)$, and movement $\left(\sigma_{\mathrm{m}}=0.05\right)$. Fig. S6 shows what happens when those three spatial scales are varied independently. Coexistence is easier to maintain if female mate search and movement are more localized (smaller $\sigma_{\mathrm{f}}$ and smaller $\left.\sigma_{\mathrm{m}}\right)$, because mating types predominating in different spatial regions then undergo less mixing. By contrast, coexistence is easier to maintain if competition occurs across a wider spatial range (larger $\sigma_{\mathrm{s}}$ ), because individuals near the resource peaks then compete more strongly for resources in the troughs, reducing population density there and thus promoting isolation of the mating types predominating near each peak.

\section{S2.8 Incorporating alternative genetic architectures}

Our main model assumes free recombination between the trait and preference loci. Fig. S8 explores the effect of linkage, finding no substantial differences between complete linkage and free recombination between the preference and display loci.

To test whether our findings are robust to changes in the number of loci, we consider a quantitative genetic model in which an individual's preference and display traits are determined by two quantitative characters. This model can be interpreted as assuming that a large (infinite) number of additive loci code for each of the two traits. Complementing our main model, which features a finite number of alleles, this 
extension allows for arbitrarily many mating types. In this quantitative genetic model, the probability that female $i$ mates with male $j$ is proportional to

$$
p_{i j}=\exp \left(-\left(p_{i}-q_{j}\right)^{2} /\left(2 \sigma_{\mathrm{p}}^{2}\right)\right) e_{i j}
$$

where $p_{i}-q_{j}$ is the difference between the preference trait of female $i$ and the display trait of male $j, \sigma_{\mathrm{p}}$ denotes the strength of female preference (smaller $\sigma_{\mathrm{p}}$ means females are choosier), and $e_{i j}$ is proportional to the encounter probability between female $i$ and male $j$, as defined in Eq. 7. Offspring trait values are drawn from a Gaussian function centred at the mean of the parental phenotypes for each trait, with a standard deviation $\sigma_{\mathrm{O}}$ that measures the variation among offspring due to segregation, recombination, and mutation. All other details of the quantitative genetic model are the same as for our main model.

Despite the different genetic assumptions, the behaviour of the quantitative genetic model closely resembles that of the allelic model (Fig. S8). Long-term coexistence of mating domains is again possible over a wide range of parameters, provided female preferences are sufficiently strong (small $\sigma_{\mathrm{p}}$ ). As in the allelic model, loss of mating domains in the quantitative genetic model, when it happens, tends to occur through the replacement of one type by the other. Compared with the allelic model, the quantitative genetic model exhibits two additional mechanisms through which mating domains may be lost. First, when female preference is weak (large $\sigma_{\mathrm{p}}$ ), interbreeding between adjacent mating domains may become so common that the resultant offspring form their own mating domains, facilitating the merging of the original domains. Second, the random drift of matched trait and preference values in one mating domain may cause them to coincide by chance with the values in an adjacent mating domain, so the two originally separate domains may merge due only to the random genetic drift of quantitative 
mating traits that results from segregation, recombination, and mutation in finite populations.

\section{S2.9 Incorporating asymmetric display costs}

Display traits can incur fitness costs in males. Our main model assumes that such costs, if present, affect all individuals equally. It may often be the case, however, that display traits differ in their effects on fitness. We therefore examine what happens when the $Q$ allele causes males to have a reduced survival probability relative to those carrying the $q$ allele (i.e., for $Q$-bearing individuals, the survival probability $s_{i}$ is reduced by a factor $1-a$, with $a$ ranging between 0 and 1). Provided that the resultant cost is not so strong that the stabilizing effect of spatial variation in local carrying capacity is overwhelmed by selection against $Q$-bearing males, our main findings remain largely unchanged (Fig. S9).

\section{References}

1. Coulson, T., Macnulty, D. R., Stahler, D. R., Vonholdt, B., Wayne, R. K., and Smith, D. W. Modeling effects of environmental change on wolf population dynamics, trait evolution, and life history. Science 334, 1275-1278 (2011).

2. Doebeli, M. and Dieckmann, U. Speciation along environmental gradients. Nature 421, 259-264 (2003).

3. Payne, R. J. H. and Krakauer, D. C. Sexual selection, space, and speciation. Evolution 51, 1-9 (1997). 


\begin{tabular}{|c|c|c|}
\hline Symbol & Eq. & Description \\
\hline \multicolumn{3}{|c|}{ Model parameters } \\
\hline$a$ & & Strength of selection against $Q$-bearing males (only S2.9) \\
\hline$k(x, y)$ & 1 & Local carrying capacity at location $(x, y)$ \\
\hline$l$ & 12 & Strength of mating-dependence in male dispersal (only S2.1) \\
\hline$m$ & 11 & Strength of mate-search costs \\
\hline$s_{\max }$ & 6 & Maximum survival probability \\
\hline$v$ & 2 & Spatial variation in local carrying capacity \\
\hline K & & Total carrying capacity \\
\hline$N$ & & Number of offspring \\
\hline$\alpha$ & 8 & Strength of female preference \\
\hline$f_{\max }$ & 9 & Maximum female fecundity \\
\hline$\lambda$ & 13 & Strength of density-dependent competition \\
\hline$\sigma_{\mathrm{f}}$ & 8 & Width of female-preference distribution \\
\hline$\sigma_{\mathrm{k}}$ & 1 & Width of peaks in local carrying capacity \\
\hline$\sigma_{\mathrm{m}}$ & & Width of movement distribution \\
\hline$\sigma_{\mathrm{o}}$ & & Width of offspring distribution (only S2.8) \\
\hline$\sigma_{\mathrm{p}}$ & 14 & Width of female preference (only S2.8) \\
\hline$\sigma_{\mathrm{s}}$ & 4 & Width of competition distribution \\
\hline \multicolumn{3}{|c|}{ Model variables } \\
\hline$c_{i}$ & 11 & Mate-search costs of female $i$ \\
\hline$d_{i j}$ & 4 & Spatial distance between individuals $i$ and $j$ \\
\hline$e_{i j}$ & 7 & Propensity for female $i$ to encounter male $j$ \\
\hline$f_{i}$ & 9 & Fecundity of female $i$ \\
\hline$n_{i j}$ & 4 & Competitive effect of individual $j$ on individual $i$ \\
\hline$p_{i j}$ & 8 & Propensity for female $i$ to choose male $j$ as a mate \\
\hline$s_{i}$ & 6 & Survival probability of individual $i$ \\
\hline$\mu_{i}$ & 10 & Local density of preferred males as seen by female $i$ \\
\hline$\rho_{i}$ & 3 & Resource share of individual $i$ \\
\hline
\end{tabular}

Table S1: Model parameters and model variables. 

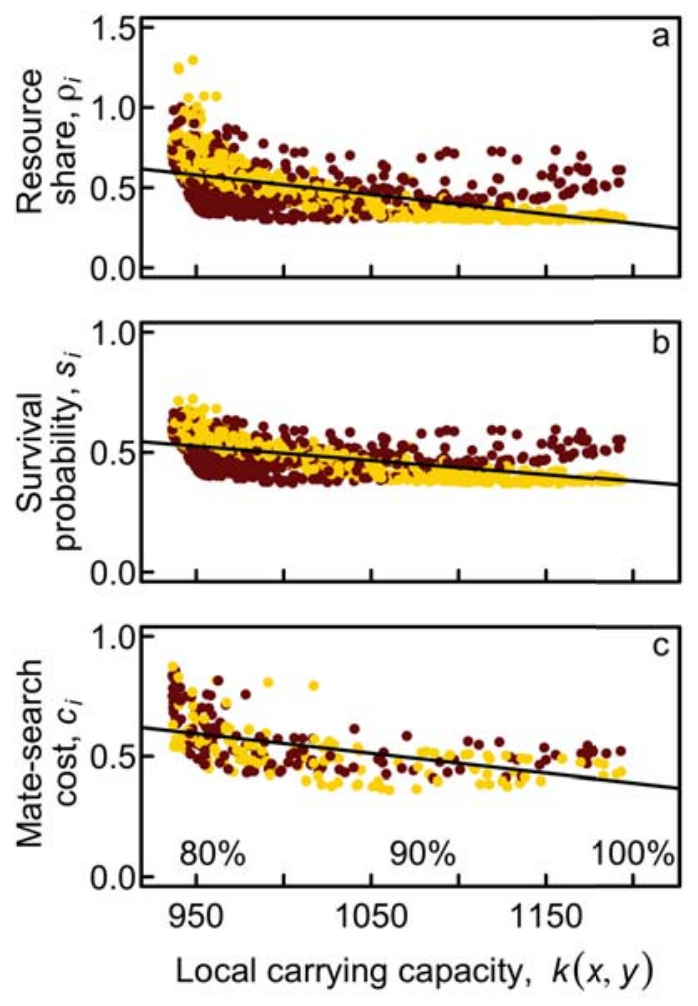

Figure S1: Variation in three components of fitness as a function of the local carrying capacity experienced by each individual at $t=1000$ for the model run in Fig. 1 d. Individuals are coloured according to their genotype at the display locus. a Resource share $\rho_{i}$ in males and females. b. Survival probability $s_{i}$ of males and females. c. Mate-search costs $c_{i}$ of females. Lines show least-squares regression lines. 


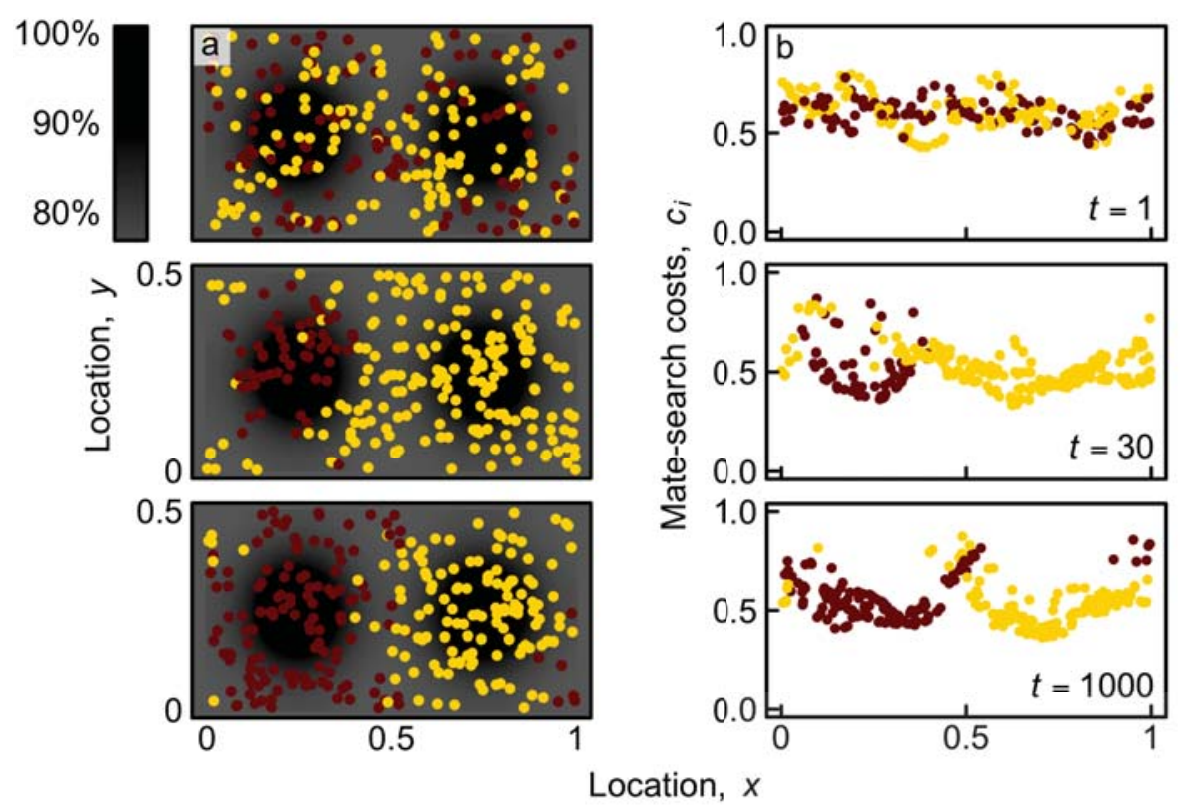

Figure S2: Mate-search costs for the model run in Fig. 1d. Panels in column a are identical to those in Fig. 1d, except that only females are shown and they are coloured according to their preference allele. Panels in column $\mathbf{b}$ show the costs associated with searching for a mate and rejecting non-preferred males for each female (Eq. 9), as a function of her location $y$. For $m / K=1$, female fecundity is typically only halved by mate-search costs. 


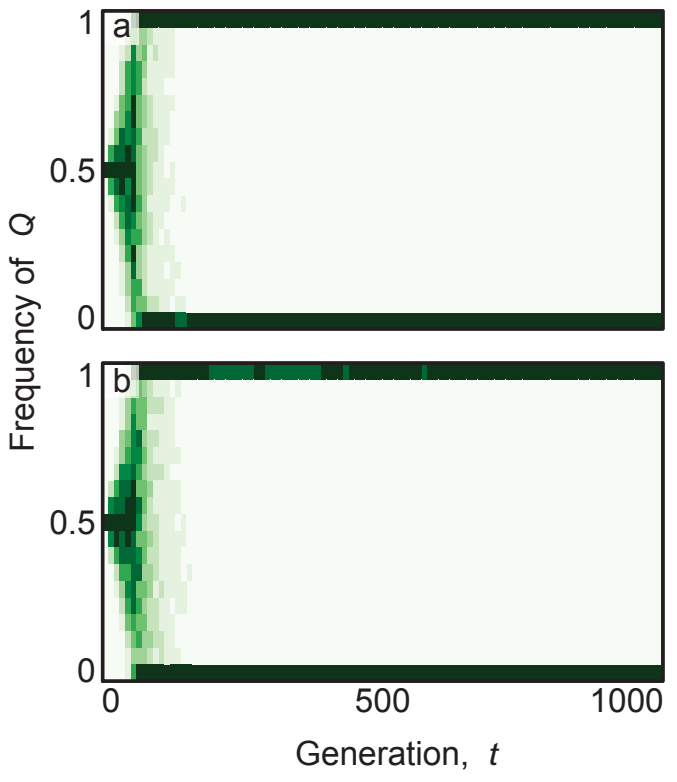

Figure S3: Effects of mating-dependent dispersal in males. Panels show distributions of allele frequencies at the display locus through time across 1000 replicate model runs in a two-dimensional homogeneous landscape; coexistence occurs only while these frequencies remain intermediate. Darker shading indicates a higher probability of observing a given frequency of the $Q$ allele. Panel $\mathbf{a}$ is identical to Fig. $2 b$. Panel $\mathbf{b}$ is the same as a, except with mating-dependent dispersal in males $(l=100)$. Results for other values of $l$ are qualitatively identical. Model runs are initialized as in Fig. 2. All other parameters are as in Fig. $1 b$. 


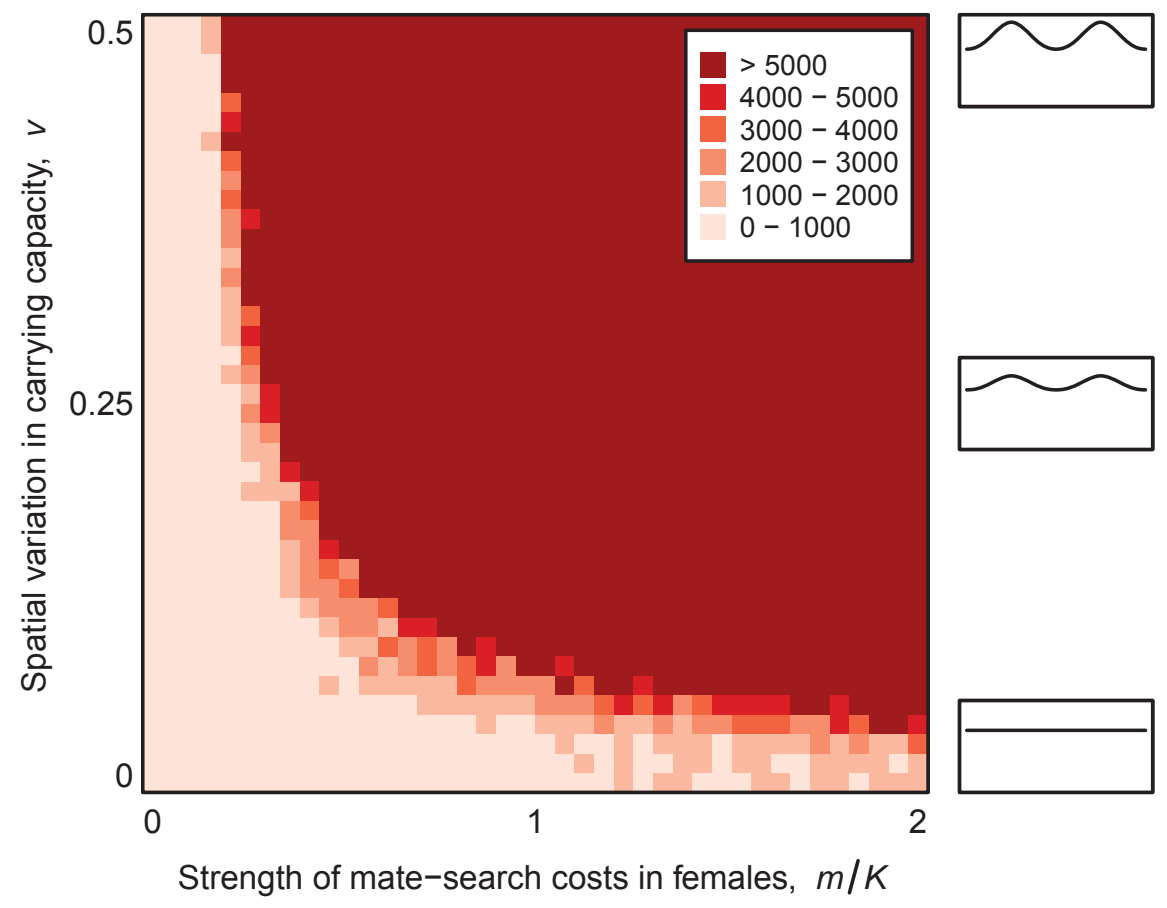

Figure S4: Conditions for long-term coexistence with competition-dependent fecundity (Section S2.3) in a two-dimensional bimodal landscape. All parameters are as in Fig. 3. 

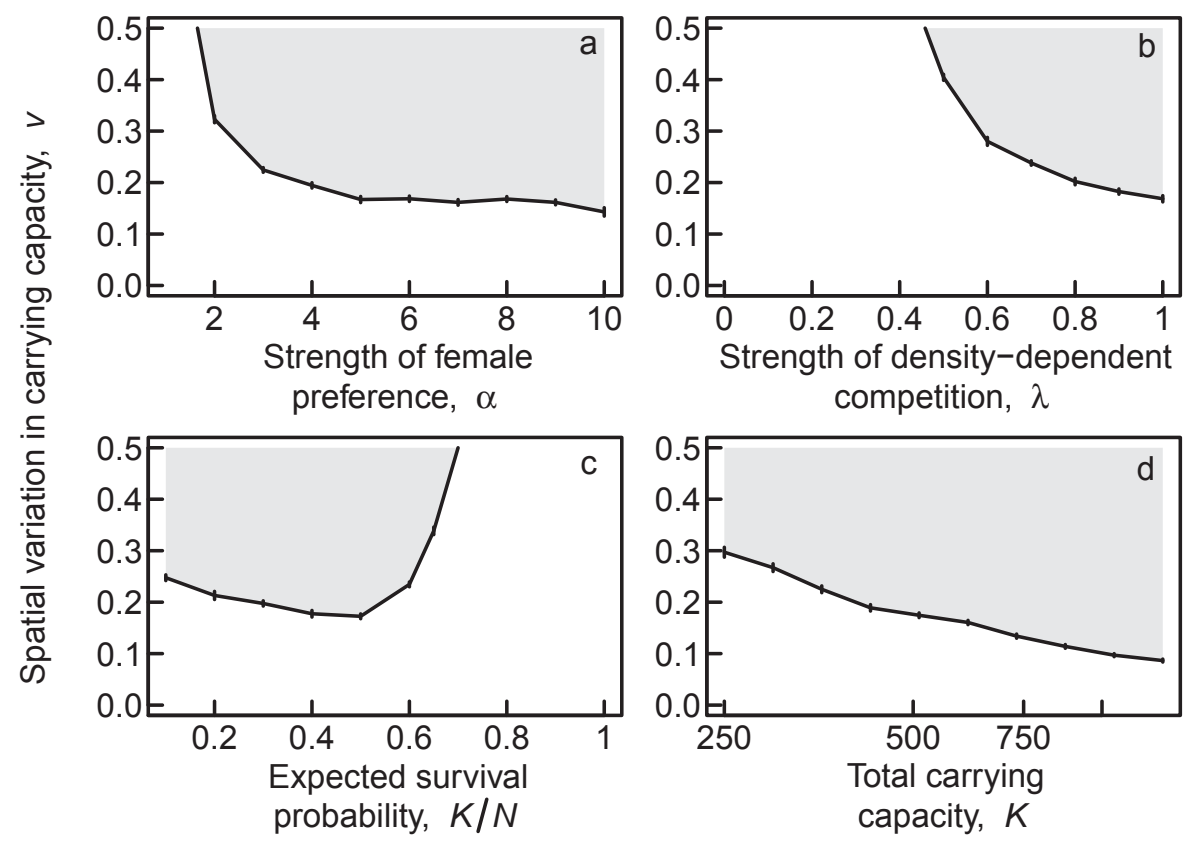

Figure S5: Minimum level of spatial variation $v$ in local carrying capacity needed to ensure long-term coexistence (grey regions) in a two-dimensional bimodal landscape. The spatial variation $v$ is increased until the average persistence time of 20 replicate runs exceeded $10 \mathrm{~K}$ generations (vertical lines indicate standard errors). a Effect of the strength $\alpha$ of female preference. Coexistence becomes more likely as female preferences become stronger (larger $\alpha$ ), although once preference exceeds $\alpha \approx 5$, its impact is small. $\mathbf{b}$. Effect of the strength $\lambda$ of density-dependent competition (varying $s_{\max }$ while holding $K=500$ and $N=1000$ constant). The limit $\lambda=0$ corresponds to completely density-independent survival, while the limit $\lambda=1$ corresponds to completely density-dependent survival. c. Effect of the expected survival probability $K / N$ (varying $N$ while holding $K=500$ and $\lambda=1$ constant). Values near $K / N=0$ correspond to very strong ecological competition, while the limit $K / N=1$ corresponds to no ecological competition. d. Effect of the total carrying capacity $K$ (varying $K$ while holding $K / N=0.5, \lambda=1$, and $m / K=1$ constant). All other parameters are as in Fig. 1d. 


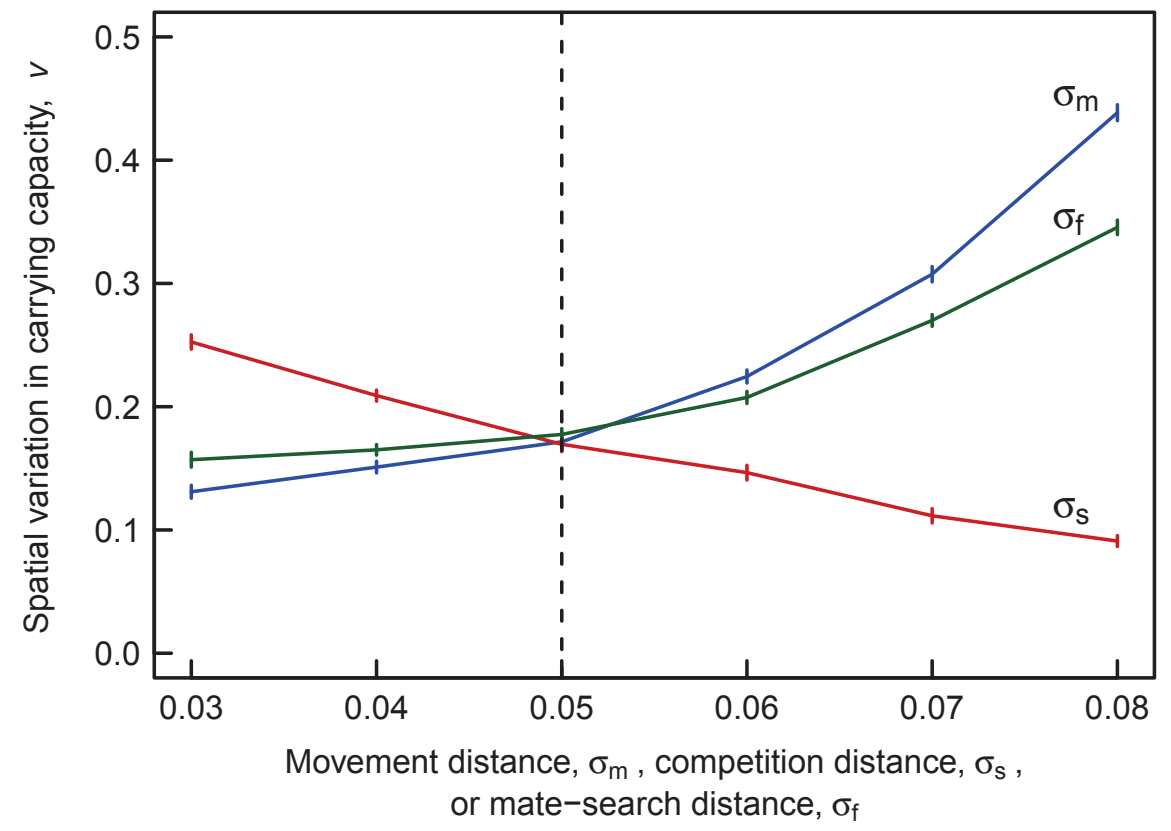

Figure S6: Minimum level of spatial variation $v$ in local carrying capacity needed to ensure long-term coexistence in a two-dimensional bimodal landscape. The spatial variation $v$ is increased until the average persistence time of mating types in 20 replicate runs exceeded $10 \mathrm{~K}$ generations (vertical lines indicate standard errors). The three curves show the effects of the width $\sigma_{\mathrm{s}}$ of the competition distribution (red), the width $\sigma_{\mathrm{f}}$ of the matesearch distribution (green), and the width $\sigma_{\mathrm{m}}$ of the movement distribution (blue), while holding all other parameters constant at their values in Fig. 1d. In the other figures, the following values (indicated by the vertical dashed line) are used: $\sigma_{\mathrm{s}}=0.05, \sigma_{\mathrm{f}}=0.05$, $\sigma_{\mathrm{m}}=0.05$. 


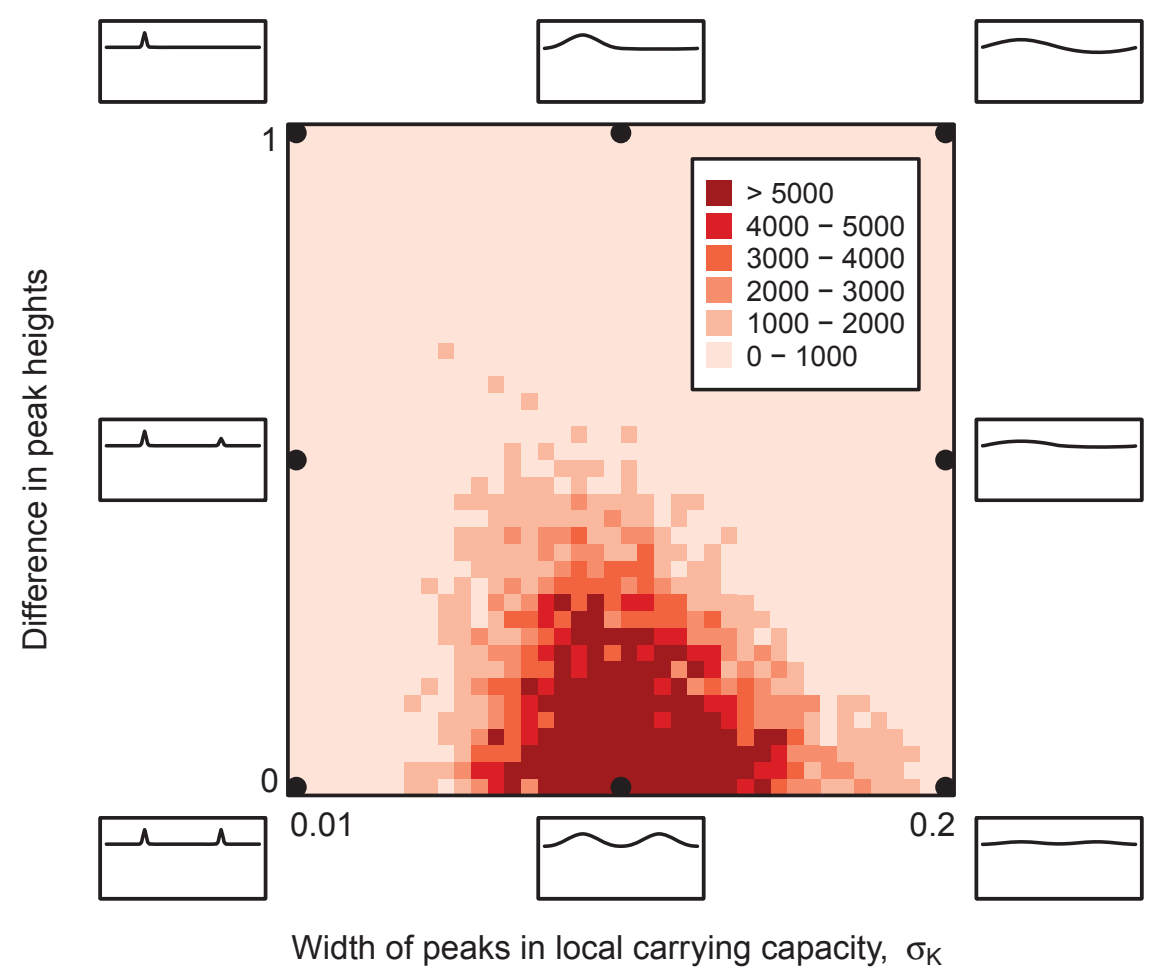

Figure S7: Effects of altering the shape of the local carrying capacity (Eq. 1) in a twodimensional bimodal landscape. Shading indicates how long polymorphism persists at the display locus $($ darker $=$ longer). Each cell represents the mean time to loss of polymorphism for 10 replicate model runs. Side panels indicate the extent of spatial variation in local carrying capacity along transects at $y=0.25$ for nine parameter combinations indicated by the closest black circle. The inset at the bottom center corresponds to the parameter combination used in Fig. 3. Spatial variation in local carrying capacity is relatively weak throughout this figure, with $v$ ranging from 0.28 for $\sigma_{\mathrm{k}}=0.01$ (far left) to 0.049 for $\sigma_{\mathrm{k}}=0.2$ (far right). All other parameters are as in Fig. $1 \mathrm{~d}$. 


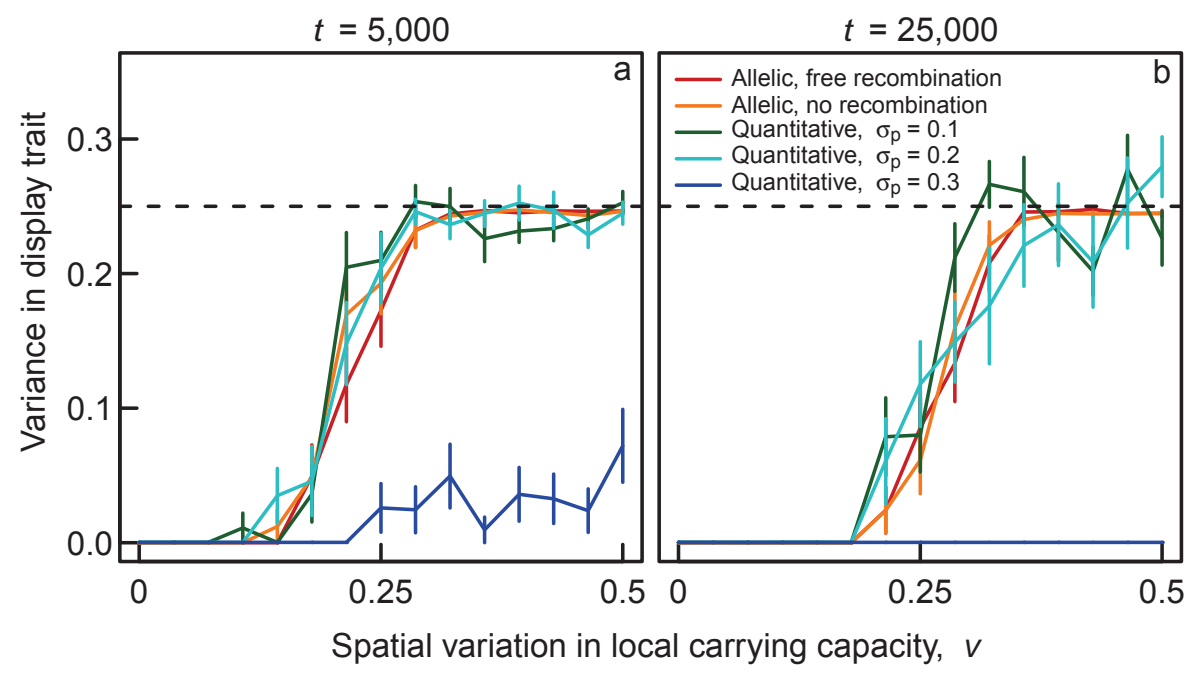

Figure S8: Effects of changes in genetic architecture in a two-dimensional bimodal landscape. Variance in display trait after 5,000 (a) and 25,000 (b) generations for a variety of genetic architectures, averaged over 20 replicate model runs (vertical lines indicate standard errors). The dashed line indicates the maximum possible variance in the allelic model (0.25). For determining variances in the allelic model, alleles $Q$ and $q$ are assigned trait values 0 and 1 , respectively. In the quantitative genetic model, the initial preference/display trait values are set to $0 / 0$ or $1 / 1$ (corresponding to $P / Q$ or $p / q$ in the allelic model) with equal probability, yielding an initial variance of 0.25 . Over time, the variance of 0.25 can be exceeded due to random genetic drift. For comparison, the red curve shows results of our main model. Model runs are initialized as in Fig. 2. All other parameters are as in Fig. 1; in the quantitative genetic model, $\sigma_{\mathrm{o}}=0.01$. 


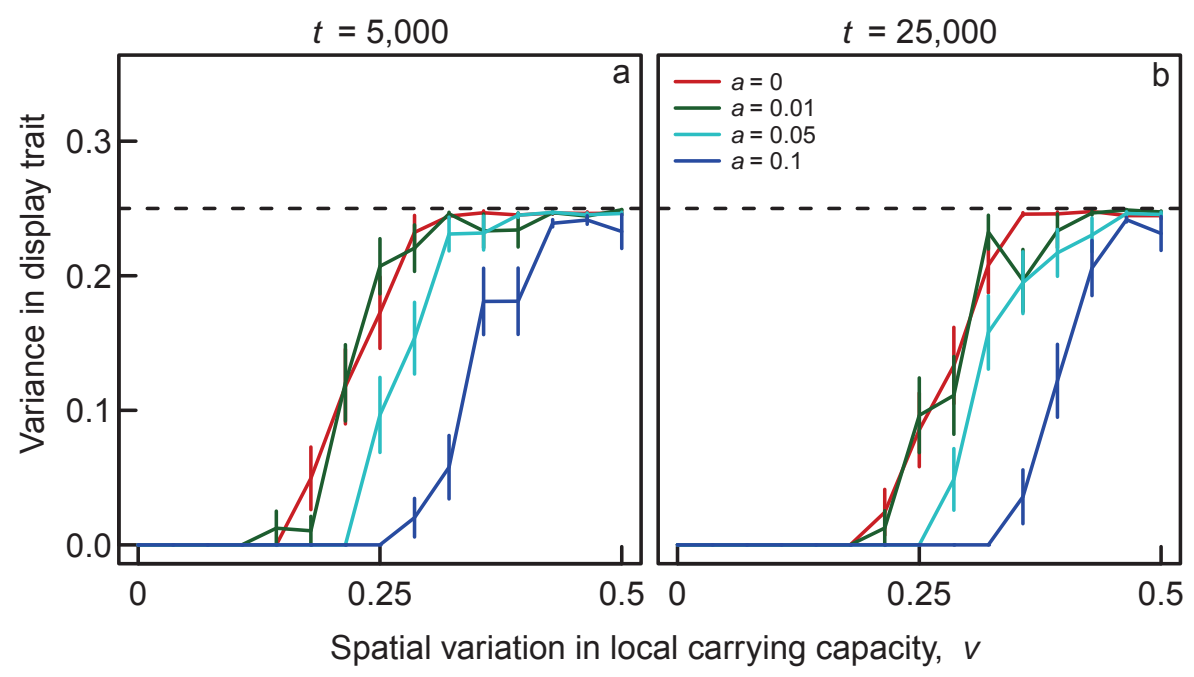

Figure S9: Effects of asymmetric fitness costs of display traits in the allelic model in a twodimensional bimodal landscape. Variance in display trait after 5,000 (a) and 25,000 (b) generations when males bearing the $Q$ allele have their survival lowered by a factor $1-a$ relative to males bearing the $q$ allele, averaged over 20 replicate model runs (vertical lines indicate standard errors). The dashed line indicates the maximum possible variance in this allelic model (0.25). For comparison, the red curve (identical to that in Fig. S8) shows results of our main model, corresponding to the limit $a=0$. Model runs are initialized as in Fig. 2. All other parameters are as in Fig. 1. 